\section{Una rara (e intermitente) masa cervical: flebectasia de la vena yugular externa}

\section{A rare (and intermittent) cervical mass: phlebectasia of external jugular vein}

Óscar Girón-Vallejo ${ }^{\mathrm{a}}$, María del Carmen Benítez-Sánchez ${ }^{\mathrm{b}}$, Vanesa Villamilc, Juan Pedro Hernández-Bermejo ${ }^{d}$

aDoctor en Medicina, Especialista en Cirugía Pediátrica, Hospital Clínico Universitario Virgen de la Arrixaca, Murcia. Hospital Virgen de la Arrixaca, Servicio de Cirugía Pediátrica, Carretera de Madrid Cartagena s/n 30120 El Palmar (MURCIA)

bDiplomada Universitaria en Enfermería. Hospital Clínico Universitario Virgen de la Arrixaca, Murcia

'Médico Interno Residente en Cirugía Pediátrica, Hospital Clínico Universitario Virgen de la Arrixaca, Murcia defe del Servicio de Cirugía Pediátrica, Hospital Clínico Universitario Virgen de la Arrixaca, Murcia

Recibido el; 10 de diciembre de 2016

Aceptado el 23 de enero de 2017

\section{Introducción}

La flebectasia de la vena yugular externa es una dilatación congénita y fusiforme del vaso que puede manifestarse como una tumoración cervical asintomática. Puede apreciarse de manera intermitente, dependiendo ello de que el paciente realice maniobras de Valsalva. El objetivo de esta comunicación es poner en conocimiento del pediatra general y de cirujanos pediátricos la existencia de una entidad clínica que por su escasa frecuencia podría pasar desapercibida o recibir tratamiento quirúrgico sin ser preciso en todos los casos.

\section{Caso clínico}

Niño de 5 años derivado a la consulta de Cirugía Pediátrica por presentar una masa cervical, de manera intermitente, apreciada por su familia desde hace unos meses. No constan antecedentes médicoquirúrgicos de interés. El paciente se encontraba asintomático. Al explorarlo, no se objetivó ninguna tumoración cervical, que sí se hizo evidente, al realizar maniobras de Valsalva (figura 1). Se le solicitó una ecografía (figura 2) y angio-RMN para completar el estudio (figura 3). Se concluyó que se trataba de una flebectasia de la vena yugular externa. Dado que el paciente está asintomático, el manejo es conservador, y tan sólo si aparecieran síntomas se plantearía el tratamiento quirúrgico.

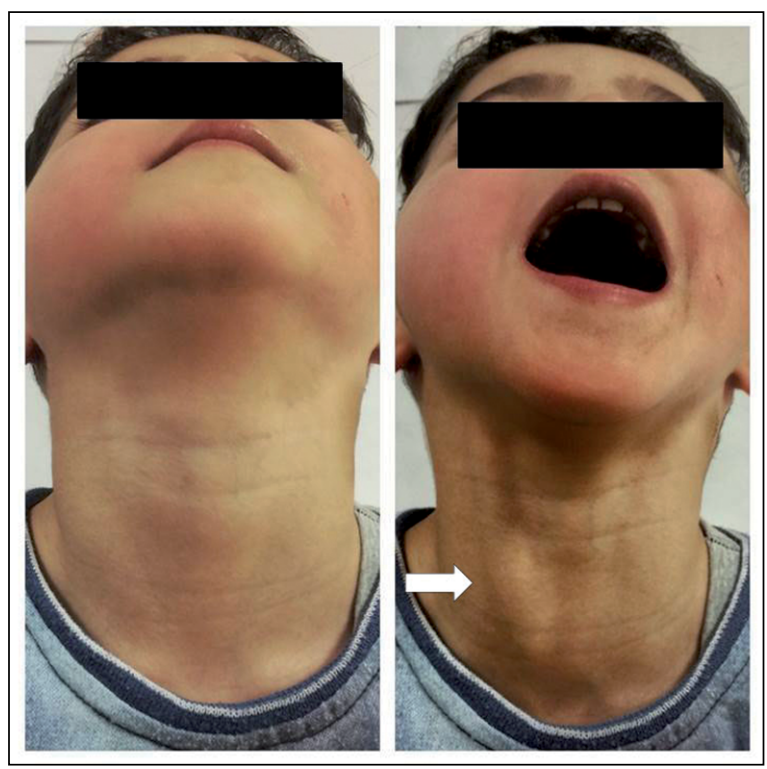

Figura 1. Exploración física del paciente en reposo y realizando una maniobra de Valsalva, donde se visualiza el aumento de volumen cervical (flecha). 


\section{Discusión}

La flebectasia es una dilatación congénita, fusiforme y aislada, sin tortuosidad, a diferencia de las varices. Clínicamente aparece como una masa blanda y compresible, no dolorosa, en el cuello de los niños al realizar maniobras de Valsalva, ya sea con el llanto o los esfuerzos. El 75\% de los casos son diagnosticados en la población pediátrica ${ }^{1}$.

Se ha sugerido la compresión de la vena innominada por la cúpula del pulmón contra la clavícula como causa de esta dilatación venosa ${ }^{2}$ y también la degeneración intrínseca de las fibras elásticas de la pared venosa. Los hallazgos histopatológicos de fibrosis difusa y arquitectura alterada del tejido elástico sugieren un mecanismo mecánico. La flebectasia es más frecuente en el varón y parecen afectarse más frecuentemente los troncos venosos del lado derecho ${ }^{3,4}$.

El diagnóstico es fundamentalmente clínico, aunque requiere conocer de forma previa esta entidad infrecuente. A pesar de tener un comportamiento benigno, puede causar gran alarma en los familiares y la confirmación del diagnóstico se realiza con ecografía doppler cervical, que identifica la dilatación venosa, el flujo venoso normal, la calidad de las paredes de la ven y la posible presencia de trombos.

El diagnóstico diferencial de las masas cervicales que aparecen intermitentemente, con las maniobras de Valsalva, es reducido. Determinados tumores o quistes y un laringocele podrían aparecer con los esfuerzos. También se ha propuesto el diagnóstico diferencial con faringocele, hernia pulmonar cervical (neumocele cervical) y malformación linfática cervical ${ }^{4}$.

Aunque algunos autores proponen la cirugía (ligadura y resección de la vena) para prevenir la trombosis o con fines cosméticos, dado que es un proceso asintomático, benigno y autolimitado, un manejo conservador parece razonable y es la opinión más generalizada, salvo en los infrecuentes casos en que asocian síntomas.

Antes de realizar la intervención quirúrgica de la flebectasia yugular, se debe comprobar la existencia de una adecuada circulación colateral mediante una angiografía con Gadolinio con resonancia magnética. Se desaconseja tratar los dos lados en el mismo acto quirúrgico, en el caso de que sea bilateral ${ }^{5}$.

\section{Conclusiones}

Ante una masa cervical de aparición intermitente, relacionada con las maniobras de Valsalva, el pediatra y el cirujano pediátrico deben pensar en la flebectasia de los troncos venosos cervicales. Asimismo, deben conocer la buena evolución en la mayoría de los casos y

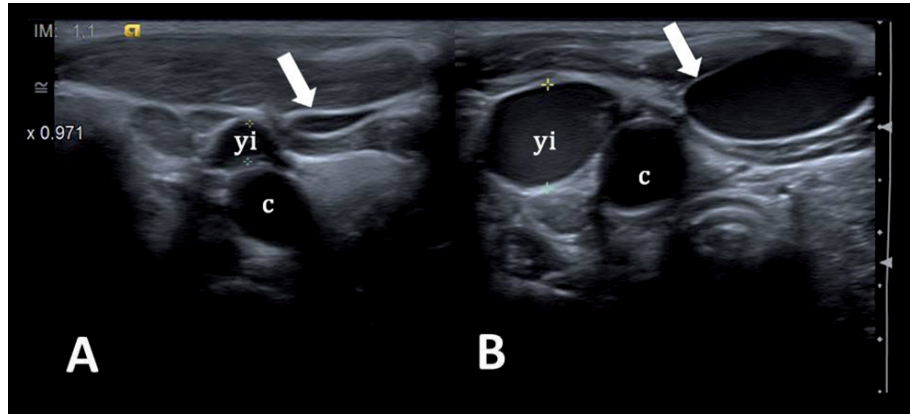

Figura 2. Ecografía de vasos cervicales: A) en reposo y B) al realizar Valsalva. Vena yugular externa derecha (flechas), yi = vena yugular interna derecha; $\mathrm{c}=$ arteria carótida derecha.

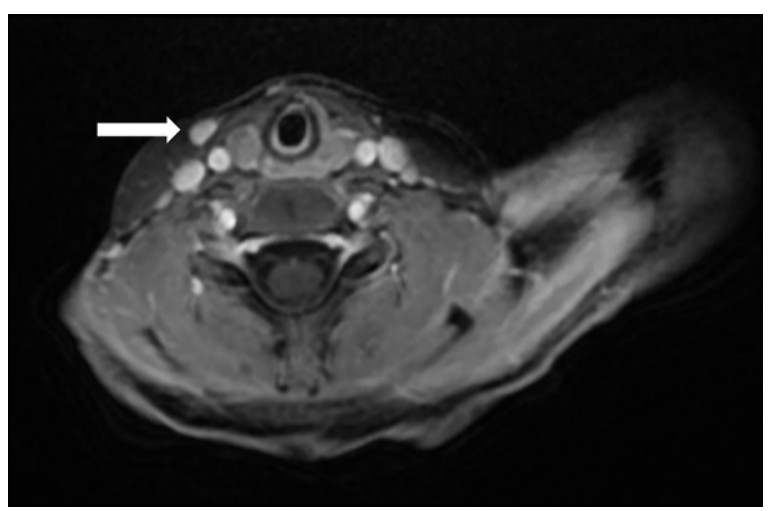

Figura 3. Angiografía mediante resonancia magnética. La flecha blanca señala la dilatación de la vena yugular externa derecha.

las escasas recomendaciones que existen en la actualidad para el tratamiento quirúrgico.

\section{Responsabilidades éticas}

Protección de personas y animales: Los autores declaran que los procedimientos seguidos se conformaron a las normas éticas del comité de experimentación humana responsable y de acuerdo con la Asociación Médica Mundial y la Declaración de Helsinki.

Confidencialidad de los datos: Los autores declaran que han seguido los protocolos de su centro de trabajo sobre la publicación de datos de pacientes.

Derecho a la privacidad y consentimiento informado: Los autores han obtenido el consentimiento informado de los pacientes y/o sujetos referidos en el artículo. Este documento obra en poder del autor de correspondencia.

\section{Conflicto de intereses}

Los autores declaran no tener conflicto de intereses. 


\section{Referencias}

1. Haney JC, Shortell CK, McCann RL, Lawson JH, Stirling MJ, Stone DH. Congenital Jugular vein phlebectasia: a case report and review of the literature. Ann Vasc Surg. 2008;22:681-3.
2. La Monte SJ, Walker EA, Noran WB. Internal jugular phlebectasia. Arch Otolaryngol. 1976;102:706-8.

3. Balik E, Erdener A, Taneli C, Mevsin A, Sayan, Yüce G. Jugular phlebectasia in children. Eur J Pediatr Surg. 1993;3:46-7.

4. Guerrero-Fernández J, Guerrero
Vázquez J, García Ascaso MT, Olmedo Sanlaureano S, Luengo Casasola JL. Flebectasia de la vena yugular interna. An Pediatr (Barc). 2005;63(1):77-88.

5. Lubianca-Neto JF, Mauri M, Prati C. Internal jugular phlebectasia in children. Am J Otolaryngol. 1999;20:415-8. 